\title{
Immune Cells Govern Cancer, Inflammation and Infections
}

\section{Zhichao Fan}

Division of Inflammation Biology, La Jolla Institute for Allergy \& Immunology, La Jolla, CA, USA

*Corresponding author: Zhichao Fan, Division of Inflammation Biology, La Jolla Institute for Allergy \& Immunology, La Jolla, CA, USA, E-mail: zhichaofan1985@gmail.com

Received date: September 18, 2017; Accepted date: September 27, 2017; Published date: September 30, 2017

Copyright: @2017 Fan Z. This is an open-access article distributed under the terms of the Creative Commons Attribution License, which permits unrestricted use, distribution, and reproduction in any medium, provided the original author and source are credited.

\section{Editor Note}

Immunobiology focuses on the biological processes in the immune system of organisms and helps us understanding and treating diseases, such as bacterial [1] and viral [2] infections, inflammation [3], cancer [4,5], auto-immune diseases [6] and chronic diseases [7]. The advanced investigations in immunobiology strive to both understand how the immune system protects our body and translate the basic immunological perspectives to clinics. Journal of Immunobiology updates the frontier advancements of immunological researches and provides perspectives in multiple fields of immunology and diseases.

The current issue of the Journal of Immunobiology presents interesting studies that could update our current knowledge. Using an in vitro system, Lin and Lin [8] investigated the effects of different natural polysaccharides on the chemokine mediated immune therapy of human breast cancer. Move from cancer to inflammation, Koga et al. [9] presented a platelet-activating factor receptor (PAFR) and signal transducer and activator of transcription 3 (STAT3) dependent signal pathway of IL-10 production by dendritic cells (DCs) during LPSstimulation. To understanding DCs better, Lin and Wang [10] briefly reviewed the roles of cytoskeletal actin and its regulators in the function of DCs, especially during the viral infections.

Breast cancer is the most frequently diagnosed malignancy in women [11]. Polysaccharides have been shown as a potential drug in treating cancers [12-14]. To investigate the killing effects of various polysaccharides on breast cancer cells, Lin and Lin [1] has purified polysaccharides from five different sources-guava, common buckwheat, bitter buckwheat, red formosa lambsquarters, and yellow formosa lambsquarters. None of these polysaccharides showed any effects on the viability and growth of breast cancer MCF-7 cells. Surprisingly, the polysaccharide-treated-splenocyte-conditioned media (SCM) showed an anti-cancer effect by inducing cell death. Cytokine measurements demonstrated that the production of IL- 2 and IL-10 by splenocytes is induced by most polysaccharides, and the ratio of IL-10 Vs. IL-2 was increased in a dose-dependent manner. Association assay suggest that the anti-cancer effect is mediated by the increase of IL-10 and IL-10/IL-2 ratio in SCM after polysaccharide stimulation. This study brings insights of immune regulation into the anti-cancer therapy by polysaccharides.

Platelet-activating factor (PAF) is a lipid mediator that is known to be involved in platelet aggregation and inflammation. PAF acts by binding to a unique $G$ protein-coupled seven transmembrane-plateletactivating factor receptor (PAFR), and activates multiple intracellular signaling pathways [15]. It is known that in dendritic cells (DC) and macrophages, exogenous PAF in conjunction with LPS induces the production of IL-10. However, the molecular mechanism remains unclear. Using inhibitors and gene editing, Koga et al. [2] investigated the mechanisms underlying the production of IL-10 upon PAFR activation. This study showed that the LPS induced the synthesis of PAF by DCs and subsequently activated PAFR. The IL-10 production was partially dependent on PAFR activation, but was independent of cAMP response element binding protein (CREB) and peroxisome proliferator-activated receptor gamma (PPAR $\gamma)$. The PAFR dependent pathway is distinct from the myeloid differentiation primary response 88 (MyD88), TIR-domain-containing adapter-inducing interferon- $\beta$ (TRIF), mitogen-activated protein kinase (MAPK) and nuclear factor kappa-light-chain-enhancer of activated B cells (NF- $\kappa \mathrm{B})$. Interestingly, the signal transducer and activator of transcription 3 (STAT3) protein was found involved in the PAFR pathway but not the other IL-10 productive pathway. This study revealed a molecular mechanism of DCs in the regulation of inflammation.

DCs are the professional antigen-presenting cells of the immune system [16]. The cytoskeletal proteins of the DCs are in involved in not only maintaining the morphology of the DCs, but are also associated with the function and maturation of the DCs. Viral infection is known to cause rearrangement of the DC cytoskeletal proteins. Regulating the behavior of the cytoskeletal proteins of DCs can be used as a therapeutic strategy for countering viral infection. Lin and Wang [3] have briefly reviewed the roles of cytoskeletons, especially actin and its mediators, in the functions of DCs. The roles and changes of actin in DCs during viral infections were also discussed.

\section{References}

1. Chen ST, Li FJ, Hsu TY, Liang SM, Yeh YC, et al. (2017) CLEC5A is a critical receptor in innate immunity against Listeria infection. Nat Commun 8: 299

2. Yu Y, Deng YQ, Zou P, Wang Q, Dai Y, et al. (2017) A peptide-based viral inactivator inhibits Zika virus infection in pregnant mice and fetuses. Nat Commun 8: 15672.

3. Fan Z, McArdle S, Marki A, Mikulski Z, Gutierrez E, et al. (2016) Neutrophil recruitment limited by high-affinity bent $\beta 2$ integrin binding ligand in cis. Nat Commun 7: 12658.

4. Enamorado M, Iborra S, Priego E, Cueto FJ, Quintana JA, et al. (2017) Enhanced anti-tumour immunity requires the interplay between resident and circulating memory CD8+ T cells. Nat Commun 8: 16073.

5. Guo J, Fan Z, Gu Z, Wei X (2012) Studying The Role of Macrophages in Circulating Prostate Cancer Cells by In Vivo Flow Cytometry. J Innov Opt Health Sci 05: 250027.

6. Fujikura D, Ikesue M, Endo T, Chiba S, Higashi H, et al. (2017) Death receptor 6 contributes to autoimmunity in lupus-prone mice. Nat Commun 8: 13957.

7. Sergin I, Evans TD, Zhang X, Bhattacharya S, Stokes CJ, et al. (2017) Exploiting macrophage autophagy-lysosomal biogenesis as a therapy for atherosclerosis. Nat Commun 8: 15750.

8. Lin HC, Lin JY (2017). Splenocyte-Conditioned Media Inhibit Breast Cancer MCF-7 Cell Growth, Associated with Increased Th2/Th1 Cytokine Secretion Ratio in the Media using Five Selected Polysaccharides. J Immuno Biol 2: 121. 
9. Koga MM, Filgueiras LR, Jancar S, Rios FJ (2017) Platelet-Activation Factor Receptor Induces Interleukin 10 Production through STAT3 Activation in Dendritic Cells. J Immuno Biol 2: 123.

10. Lin W, Wang X (2017) Regulating DCs in Innate Immune Response and Infection by Cytoskeletal Proteins. J Immuno Biol 2: 124

11. Siegel RL, Miller KD, Jemal A (2017) Cancer statistics, 2017. CA Cancer J Clin 67: 7-30.

12. Zong A, Cao H, Wang F (2012) Anticancer polysaccharides from natural resources: a review of recent research. Carbohydr Polym 90: 1395-1410.
13. Lemieszek M, Rzeski W (2012) Anticancer properties of polysaccharides isolated from fungi of the Basidiomycetes class. Contemp Oncol (Pozn) 16: 285-289.

14. Xu H, Xu X (2016) Polysaccharide, a Potential Anti-Cancer Drug with High Efficacy and Safety. J Oncol Res Treat 2: 110

15. Ishii S, Nagase T, Shimizu T (2002) Platelet-activating factor receptor. Prostaglandins Other Lipid Mediat 68-69: 599-609.

16. Lin W, Fan Z (2016) Immunological Synapse Molecules. J Immuno Biol 1: 111. 